\title{
L'IMPLICATION DES ACTEURS DE LA RECHERCHE DANS LES PROCESSUS D'ADAPTATION AU CHANGEMENT CLIMATIQUE : L'EXEMPLE DES RÉGIONS VITICOLES FRANÇAISES
}

\author{
James BOYER \\ INRA-Supagro \\ UMR Innovation, Université de Montpellier \\ james.boyer@supagro.inra.fr
}

Le réchauffement climatique est aujourd'hui un défi majeur pour l'environnement et les activités économiques, interrogeant non seulement la capacité des acteurs économiques à formuler des stratégies pertinentes, mais aussi la capacité des scientifiques à produire de nouvelles connaissances pour faire face aux conséquences du changement climatique (CC). Ce besoin de connaissances scientifiques se justifie par la recherche d'une meilleure compréhension et anticipation du phénomène, alors que les perceptions individuelles des effets du changement climatique sont limitées dans le temps et dans l'espace. Il se justifie également par la nécessité de limiter les incertitudes sur les scénarios d'évolution du climat à différentes échelles (Le Treut, 2003), sur les réactions des écosystèmes (Cramer et al., 2001), et sur les pistes d'adaptation possibles pour les agents économiques et la société dans son ensemble (Smit, Wandel, 2006 ; Stern, Treasury, 2006 ; Füssel, 2007).

Le secteur viticole, précisément, est fortement marqué par le CC et son adaptation à l'évolution climatique constitue un enjeu majeur au niveau mondial et en France (Ollat, Touzard, 2014). Les impacts du réchauffement climatique sont en effet déjà visibles dans les vignobles français et menacent le niveau de production du vin, la typicité du terroir ou même le système d'appellation d'origine (très liés aux spécificités pédoclimatiques) lequel structure le secteur viticole français et construit la singularité et la réputation des vins français (Goutouly, 2009 ; Ollat, Touzard, 2014). En effet, des 
travaux utilisant des modèles climatologiques prévoient que la plupart des régions vitivinicoles françaises pourraient enregistrer une diminution sensible du niveau de production, principalement les régions méditerranéennes dont la baisse pourrait atteindre jusqu'à $75 \%$ (Hannah et al., 2013). Les effets les plus visibles sont, entre autres, l'avancée des dates de vendanges, des stress hydriques plus prononcés pour la vigne, l'augmentation du taux d'alcool et la diminution de l'acidité du vin (Schultz, 2000 ; Jones, 2007 ; Duchêne et al., 2010). Les viticulteurs innovent déjà pour s'adapter, principalement à partir de leurs expériences dans la gestion de la variabilité climatique (Jouan, 2014). Cependant, les nouveaux enjeux que pose la problématique climatique interrogent la pertinence des connaissances empiriques actuelles (prise en compte du long terme, compromis entre adaptation et mitigation, liens avec les stratégies spatiales et les changements institutionnels, nature des connaissances mobilisées et rôle de la recherche). La recherche scientifique est alors sollicitée pour traiter une large gamme de questions, de manière spécifique ou transversale, sur les possibilités d'innovation pour s'adapter aux nouvelles conditions climatiques. Se pose alors la question de l'implication réelle des chercheurs dans la construction et diffusion de nouvelles connaissances en vue de renforcer les capacités d'adaptation de la viticulture au CC.

Dans le cadre d'une approche en termes de "capacité d'adaptation », nous voulons tester l'idée que l'implication des chercheurs dépend de leur investissement ${ }^{1}$ dans la production de connaissances autour du CC, mais aussi de la nature des liens qu'ils construisent avec les acteurs des entreprises du secteur. Nous chercherons à expliquer cette implication en analysant les facteurs qui renvoient à la trajectoire et aux caractéristiques personnelles du chercheur et à des facteurs plus systémiques comme sa région d'exercice et le type d'organisme auquel il est rattaché.

Nous expliciterons en premier lieu le cadre théorique des approches en termes de «capacité d'adaptation » pour étudier le rôle des connaissances dans l'adaptation au CC, puis nous présenterons notre méthodologie d'enquête et d'analyse. Nous décrirons alors nos principaux résultats que nous discuterons au regard de travaux récents sur les Systèmes Sectoriels d'Innovation qui visent à analyser les relations entre les acteurs, institutions, organisations et réseaux impliqués dans le processus d'innovation au niveau d'un secteur donné (Malerba, 2002 ; Lorius, 2003).

1. Le mot investissement est employé dans cet article pour exprimer le fait, pour un individu, de mettre beaucoup de lui-même dans une action ou un travail. 


\section{CAPACITÉ D’ADAPTATION AU CHANGEMENT CLIMATIQUE}

L'hypothèse générale de notre travail est que plus les acteurs économiques disposent de ressources cognitives sur l'évolution climatique et ses impacts (connaissances disponibles, accessibilité à l'information), plus ils sont capables de s'adapter au CC. Les incertitudes qui entourent ces phénomènes nous amènent alors à mobiliser les travaux qui conçoivent l'adaptation sous l'angle des « capacités » (Smit, Wandel, 2006), en se focalisant sur la construction de bases de connaissances (Leary et al., 2006) et des relations entre scientifiques et acteurs économiques d'un secteur (Vescovi et al., 2009).

\section{Adaptation au changement climatique : approche par les capacités}

L'approche de l'adaptation capacity en rapport avec le CC a été développée récemment pour prendre en compte la manière dont les agents économiques peuvent mieux gérer les incertitudes liées à l'évolution climatique et leur vulnérabilité face à ce phénomène (Smit, Pilifosova, 2003 ; Smit, Wandel, 2006). En effet, il ne suffit pas de mettre en place des stratégies vues comme optimales à un moment donné pour répondre aux effets " attendus » du CC. Ladaptation suppose d'être capable d'accompagner l'incertitude quitte à changer de stratégies et de trajectoire si nécessaire, en fonction de l'évolution des connaissances (IPCC, 2001).

La capacité d'adaptation au CC dépend d'un ensemble de facteurs tels que les ressources cognitives, économiques et technologiques, la capacité de gestion, la qualité des infrastructures, le capital institutionnel et le capital relationnel (Smit, Pilifosova, 2003 ; Smit, Wandel, 2006 ; Rodrigo, 2015). La prise en compte de la dimension spatiale du CC constitue aussi un paramètre important pour mettre en cohérence la production de connaissances avec les enjeux locaux. En effet, la capacité adaptative permet aux entreprises ou aux agents économiques d'être réactifs par rapport à la dynamique climatique et à sa complexité. Elle les rend apte à explorer une pluralité de voies et d'innovations possibles et à choisir des actions optimales pour répondre aux conséquences du CC à court, moyen ou long terme.

\section{Bases de connaissances et capacité d'adaptation}

Les connaissances scientifiques jouent en effet un rôle clé dans l'adaptation au CC. Schipper et Burton (2009) ont montré que l'insuffisance de «bases de connaissances de l'adaptation » constitue un goulot 
d'étranglement qui limite les réponses à l'évolution climatique (Schipper, Burton, 2009). Les agents économiques sont souvent incertains ou sceptiques sur les effets du CC, les causes qui y contribuent, la probabilité d'occurrence de ces événements et leurs conséquences pour le développement économique (Leary et al., 2006).

Les acteurs de la recherche et de l'expérimentation sont donc interpellés par le CC, car cet enjeu bouleverse les connaissances actuelles et suscite de nouveaux problèmes qui appellent à élargir les champs de connaissances, à produire de nouvelles formes de connaissances en reliant des domaines scientifiques qui étaient éloignés les uns des autres (économie-climatologie, politique-climatologie, etc.) et à renforcer le lien entre les dimensions globale et locale des problèmes.

Certains travaux plaident pour une articulation efficiente des connaissances scientifiques avec des connaissances traditionnelles, écologiques et locales (Vescovi et al., 2009 ; Santha et al., 2010 ; Boyer et al., 2014). En effet, il a été démontré empiriquement que ce dernier type de connaissances influence les perceptions des communautés sur le CC et les réponses qu'elles apportent aux changements environnementaux (Nyong et al., 2007 ; Reedy et al., 2014). Il faut alors questionner d'un côté l'accessibilité des entreprises aux connaissances scientifiques et, de l'autre côté, le processus de partage d'expériences et des pratiques avec les acteurs de la recherche, qui pourrait se concrétiser par la création de groupes consultatifs entre les scientifiques et les acteurs économiques, sur l'impact des aléas climatiques et sur des pistes d'adaptation (Pennesi et al., 2012).

\section{Réseaux d'adaptation et capacité d'adaptation}

L'enjeu du CC appelle aussi les chercheurs à reconsidérer leur rôle dans le système économique qui, au-delà de la production de connaissances, interroge leur capacité à s'engager dans la diffusion de connaissances de base susceptibles de renforcer la capacité des agents économiques et politiques à identifier, développer et mettre en œuvre des réponses efficaces (Rosenzweig, Wilbanks, 2010 ; Ghini et al., 2012). Cela suggère le renforcement des relations science-industrie dans leurs différentes modalités (relations interpersonnelles entre scientifiques et entrepreneurs, liens à partir des projets publics-privés, consultations, formation de cadres qualifiés que vont recruter les entreprises, échanges d'informations via des réunions et des conférences...) (Cohen et al., 2002 ; Giuliani, Bell, 2005 ; D’Este, Patel, 2007).

Une «faible importance» des réseaux entre scientifiques et acteurs économiques semble nuire à l'accessibilité des connaissances et au partage 
d'expériences sur l'adaptation, empêchant ces derniers d'obtenir les informations nécessaires pour prendre des décisions pertinentes (Vescovi et al., 2009). Le renforcement de la capacité des acteurs à identifier, à documenter et à diffuser les meilleures pratiques, à apprendre des expériences émergentes, constitue un véritable défi pour les programmes de recherche et de développement. Des travaux scientifiques suggèrent de construire des réseaux et des partenariats à long terme entre les universitaires, les praticiens et d'autres intervenants des communautés, pour générer, communiquer et appliquer les connaissances en vue de prendre de bonnes décisions et de gérer efficacement les risques climatiques (Leary et al., 2006 ; Grin, 2010).

\section{Réseaux, connaissances et capacité d'adaptation dans l'industrie du vin}

Plusieurs facteurs d'ordre technique, économique, social et institutionnel sont susceptibles d'influencer la capacité d'adaptation de l'industrie de la vigne et du vin. Suivant la région viticole, ces facteurs sont de nature et d'importance variables (Hinnewinkel, 2007 ; Holland, Smit, 2010). Les ressources cognitives sont repérées comme déterminantes dans la réponse à la problématique climatique (Ollat, Touzard, 2014). La densité et surtout la structure des « réseaux professionnels » sont également une composante constitutive de clusters favorisant l'innovation dans la viticulture (Giuliani, Bell, 2005 ; Giuliani, Arza, 2009). Ces connaissances et réseaux doivent aussi se raisonner en fonction du domaine ou de l'objet sur lequel porte l'innovation ou la recherche (Chiffoleau, Touzard, 2014). Au moins cinq domaines de recherche sont ainsi concernés par la question climatique :

- Le matériel végétal, c'est-à-dire les potentialités des variétés de vigne cultivées (les cépages disponibles) ou créées (hybrides notamment) pour faire face aux conséquences du CC (Van Leeuwen et al., 2013) ;

- Les modes de conduite du vignoble (architecture des vignes, itinéraires techniques, gestion de la contrainte hydrique...) adaptés au CC, par exemple pour favoriser la fraicheur au niveau des raisins, limiter l'évapotranspiration ou faire face au déficit hydrique (Goutouly, 2009; Carbonneau, Ojeda, 2012);

- La lutte contre la pression parasitaire, provoquée notamment par les nouvelles conditions climatiques, à travers la veille et des nouvelles méthodes de traitement ou de contrôle des parasites (Quénol, 2014);

- Les pratiques oenologiques qui peuvent corriger les effets du CC sur la qualité du vin (équilibre du profil aromatique, degré alcoolique et acidité) (Kontoudakis et al., 2011) ; 
- Les stratégies spatiales et économiques, interrogeant les décisions des producteurs à s'installer dans une zone de production par rapport aux scénarios localisés du CC (potentialités biophysiques et hydrographiques des zones de production) (Schultz, Stoll, 2010), mais aussi les coûts et les bénéfices des stratégies d'adaptation à différentes échelles.

\section{MÉTHODOLOGIE}

Notre méthodologie se base sur une enquête face-à-face auprès d'acteurs de la recherche et de l'innovation localisés dans différents vignobles. Nous caractérisons l'« implication» de ces acteurs à s'adapter au CC à partir de deux composantes : leur investissement dans la production de connaissances pour l'adaptation et leur proximité avec les acteurs économiques du secteur. Nous mettons en relation des indicateurs de ces deux composantes avec des variables qui peuvent les "expliquer » et renvoient aux caractéristiques et à la trajectoire personnelles du chercheur, à son excellence académique, à son domaine de recherche, à son organisation de rattachement et à sa région d'exercice.

\section{Choix des régions viticoles}

Trois vignobles régionaux ont été retenus: Champagne, LanguedocRoussillon et Aquitaine (Bordeaux). Ces vignobles représentent trois aires climatiques distinctes. Le Languedoc-Roussillon, en zone méditerranéenne, est caractérisé par un climat doux tout au long de l'année, une pluviométrie relativement faible, des étés chauds, des hivers secs avec des vents qui apportent souvent de l'air froid. L'Aquitaine, sous influence océanique, a une pluviométrie beaucoup plus abondante, mais surtout bien repartie toute l'année, des hivers doux, des étés secs mais frais. La Champagne, en zone plus continentale et septentrionale, est marquée par une amplitude thermique plus importante avec des étés très chauds, des hivers rudes, une pluviométrie assez importante en été avec des pluies orageuses et, en hiver, des précipitations sous forme de neige. Les scénarios climatiques diffèrent également pour ces trois régions. Certains modèles prévoient à l'horizon 2050 une diminution de $67 \%$ des surfaces « favorables » à la production de vin de qualité en Languedoc-Roussillon et Aquitaine (Hannah et al., 2013). Par contre, la Champagne serait davantage épargnée. En Languedoc-Roussillon et à Bordeaux, sont prévus des baisses de rendement, des vins plus alcoolisés et moins acides. Pour le vignoble champenois, les modèles climatiques prévoient des canicules estivales (comme celle de 2003) et des gels de printemps plus fréquents (Briche, 2011). 


\section{Choix des organisations et acteurs enquêtés}

Une première série d'entretiens exploratoires et la consultation de documents institutionnels ont permis de préciser les organismes, unités de recherche ou services impliqués dans la production et la diffusion de connaissances scientifiques et techniques sur la vigne et le vin : centres de recherche (INRA, CNRS, IRSTEA), universités des 3 régions, écoles d'ingénieurs (Montpellier SupAgro, Bordeaux Sciences Agro) et principaux organismes de $R \& D$ associés $\left(I^{2} V^{2}\right.$, services viticoles des Chambres d'Agriculture $^{3}$, interprofessions ${ }^{4}$ ). Des listes de chercheurs, enseignantschercheurs, ingénieurs et techniciens ont été établies pour l'ensemble des unités et services de ces organismes. Les enquêtes ont été réalisées auprès d'un échantillon raisonné de ces acteurs, par tirage aléatoire par région et par organisme (voir Tableau 1). Les 94 enquêtes ont été effectuées en face-àface de mai à novembre 2014.

Tableau 1 - Répartition des personnes enquêtées dans les organismes

\begin{tabular}{|c|c|c|c|c|}
\hline RÉGION & & Organisme & Échantillon & Estimation de la population \\
\hline \multirow{4}{*}{ Bordeaux } & \multirow{3}{*}{ Experim } & $\begin{array}{l}\text { Chambre } \\
\text { d'Agriculture }\end{array}$ & 5 & 50 \\
\hline & & IFV & 4 & 15 \\
\hline & & CIVB & - & 5 \\
\hline & Chercheurs & Recherche/université & 25 & 200 \\
\hline \multicolumn{3}{|l|}{ Total } & 34 & 270 \\
\hline \multirow{4}{*}{ Champagne } & \multirow[t]{3}{*}{ Experim } & $\begin{array}{l}\text { Chambre } \\
\text { d'Agriculture }\end{array}$ & 4 & 7 \\
\hline & & IFV & - & 4 \\
\hline & & CIVC & 8 & 43 \\
\hline & Chercheurs & Recherche/université & 7 & 70 \\
\hline \multicolumn{3}{|l|}{ Total } & 19 & 124 \\
\hline \multirow{4}{*}{$\begin{array}{l}\text { Languedoc- } \\
\text { Roussillon }\end{array}$} & \multirow{3}{*}{ Experim } & $\begin{array}{l}\text { Chambre } \\
\text { d'Agriculture }\end{array}$ & 4 & 50 \\
\hline & & IFV & 7 & 15 \\
\hline & & CIVR/CIVL & - & 5 \\
\hline & Chercheurs & Recherche/université & 30 & 220 \\
\hline \multicolumn{3}{|l|}{ Total } & 41 & 290 \\
\hline \multicolumn{3}{|c|}{ Total général } & 94 & 684 \\
\hline
\end{tabular}

Source : J. Boyer, 2015

2. L'IFV est un institut technique qui a des pôles présents dans les différentes régions viticoles. 3. Les Chambres d'Agriculture sont des organisations de conseil, d'expérimentation et de développement agricole organisées au niveau régional et départemental.

4. Les interprofessions vitivinicoles sont des organisations qui regroupent les producteurs et le négoce. Elles ont parfois un service technique qui fait de l'expérimentation. 
Les acteurs enquêtés ont été catégorisés en deux groupes selon leur activité et appartenance institutionnelle. Le groupe 1 concerne les chercheurs et enseignants-chercheurs, principaux producteurs de connaissances scientifiques et évalués selon leurs publications ; le groupe 2 rassemble les acteurs d'organismes techniques, évalués au regard de leurs missions d'expérimentation ou de développement. Nous qualifierons le premier groupe de "chercheurs » et le second d' « expérimentateurs ». Les acteurs de ces deux groupes seront appelés « acteurs de la $R \& D$ ».

\section{Analyse des données}

Pour déterminer l'implication des acteurs de la $R \& D$ dans l'adaptation de la filière vitivinicole nous avons analysé (Tableau 2) :

1. L'investissement des acteurs de la $R \& D$ dans la production des connaissances sur le CC en leur demandant de situer la place de la thématique CC dans leurs activités, selon une échelle de 1à 5 (1 si l'acteur ne travaille pas sur le CC, 5 si le CC est son axe de recherche principal).

2. La proximité des acteurs de $R \& D$ avec les viticulteurs, responsables de domaines ou de caves coopératives a été évaluée à partir des relations directes et de conseil. Les liens de conseil renvoient à des échanges répétés, des discussions, des informations données avec une certaine confiance. Nous avons utilisé comme proxy le nombre de chefs d'entreprises vitivinicoles cités par les acteurs de la $R \& D$, appelé « nombre de contacts».

Nous avons croisé ces variables avec des facteurs susceptibles de les expliquer, issus également des enquêtes (Tableau 3) :

1. Les caractéristiques et la trajectoire personnelles décrites par le sexe, l'âge, l'expérience à l'étranger et la participation à des projets de recherche ou d'expérimentation sur le CC.

2. L'excellence académique composée par le niveau d'étude et le nombre des publications scientifiques du chercheur.

3. Nous avons retenu cinq domaines de recherche ou d'action, cités plus haut, constituant des leviers d'adaptation au CC.

4. La Région: Aquitaine-Bordeaux, Languedoc-Roussillon ou Champagne.

5. L'organisme de rattachement, qui peut être un centre de recherche ou une université/école d'ingénieur, l'IFV, une Chambre d'Agriculture ou une interprofession. 


\section{Tableau 2 - Variables dépendantes}

\begin{tabular}{|c|c|c|}
\hline 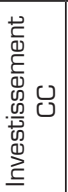 & $\begin{array}{l}\text { ICC : acteurs de la R\&D } \\
\text { ICCR : chercheurs seuls }\end{array}$ & $\begin{array}{l}\text { Variable d'échelle qui mesure l'investissement de l'acteur de la } \\
\text { recherche au CC. Elle prend la valeur } 1 \text { s'il ne travaille pas du } \\
\text { tout sur le CC, } 2 \text { si il travaille indirectement, } 3 \text { si cet enjeu est } \\
\text { présent dans ses recherches, } 4 \text { si c'est l'un des principaux } \\
\text { axes de recherche, } 5 \text { si c'est le principal axe de recherche }\end{array}$ \\
\hline 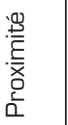 & $\begin{array}{l}\text { CAVIX : acteurs de la } \\
\text { recherche } \\
\text { CAVIR : Chercheurs seuls }\end{array}$ & $\begin{array}{l}\text { Variable numérique qui mesure le nombre d'entreprises } \\
\text { viticoles (coopérative, domaine) avec lesquelles l'acteur de la } \\
\text { recherche échange régulièrement }\end{array}$ \\
\hline
\end{tabular}

\section{Tableau 3 - Les variables indépendantes}

\begin{tabular}{|c|c|c|}
\hline \multirow{8}{*}{ 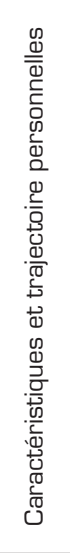 } & NAR & $\begin{array}{l}\text { Variable quantitative qui mesure le nombre de publications dans des revues } \\
\text { scientifiques du chercheur }\end{array}$ \\
\hline & FORM & $\begin{array}{l}\text { Variable d'échelle qui exprime le niveau d'étude de l'acteur. Elle prend la valeur } \\
1 \text { s'il est au niveau BAC+5 ou moins, } 2 \text { si doctorat et } 3 \text { si HDR }\end{array}$ \\
\hline & INTERN & $\begin{array}{l}\text { Variable dichotomique qui prend la valeur } 1 \text { si le chercheur a eu une } \\
\text { expérience internationale et } 0 \text { sinon }\end{array}$ \\
\hline & ANC & $\begin{array}{l}\text { Variable quantitative qui mesure l'expérience du chercheur à savoir le nombre } \\
\text { d'années depuis qu'il travaille dans le secteur vitivinicole }\end{array}$ \\
\hline & AGE & Variable quantitative qui estime l'âge du chercheur \\
\hline & SEXE & $\begin{array}{l}\text { Variable dichotomique qui prend la valeur } 1 \text { si le chercheur est de sexe } \\
\text { masculin et } 0 \text { sinon }\end{array}$ \\
\hline & PRO & $\begin{array}{l}\text { Variable dichotomique qui prend la valeur } 1 \text { si l'acteur a participé à un projet } \\
\text { de recherche sur le CC et } 0 \text { sinon }\end{array}$ \\
\hline & RESO & $\begin{array}{l}\text { Variable dichotomique qui prend la valeur } 1 \text { si l'acteur, dans le cadre de ses } \\
\text { activités professionnelles, travaille avec au moins un réseau formel d'acteurs } \\
\text { professionnels et } 0 \text { sinon }\end{array}$ \\
\hline 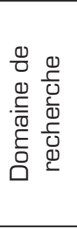 & DREC & $\begin{array}{l}\text { Variable catégorielle qui comporte } 5 \text { catégories } \\
\text { - Eno = si l'acteur travaille dans le domaine de l'œenologie ; } \\
\text { - Stratloc = si l'acteur travaille dans le domaine des stratégies spatiales et } \\
\text { économiques ; } \\
\text { - Condt = si l'acteur travaille dans le domaine de la conduite du vignoble ; } \\
\text { - Lut = si l'acteur travaille dans le domaine de luttes contre les maladies; } \\
\text { - mat = si l'acteur travaille dans le domaine du matériel végétal. }\end{array}$ \\
\hline 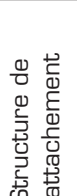 & STRUC & $\begin{array}{l}\text { Variable catégorielle qui comporte } 4 \text { catégories } \\
\text { - Strucf= si l'acteur est rattaché à des centres de recherches ou des } \\
\text { universités ; } \\
\text { - IFV = si l'acteur est rattaché à IFV ; } \\
\text { - } \quad \mathrm{CA}=\text { si l'acteur est rattaché à une Chambre d'Agriculture ; } \\
\text { - INTERPRO = si l'acteur est rattaché à l'interprofession. }\end{array}$ \\
\hline & STRUCF & $\begin{array}{l}\text { Variable dichotomique qui prend la valeur } 1 \text { si l'acteur est attaché à un centre } \\
\text { de recherche ou une université et } 0 \text { sinon }\end{array}$ \\
\hline 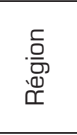 & REG & $\begin{array}{l}\text { Variable catégorielle qui comporte } 3 \text { catégories } \\
\text { - } \quad \text { LR }=\text { si l'acteur est localisé au niveau du Languedoc Roussillon ; } \\
\text { - } \quad \text { Bx = si l'acteur est localisé au niveau de Bordeaux; } \\
\text { - } \quad \text { Ch }=\text { si l'acteur est localisé au niveau de Champagne. }\end{array}$ \\
\hline
\end{tabular}

Source : J. Boyer, 2015 


\section{Modèles économétriques 5}

L'investissement de l'acteur de $R \& D$ dans la production de connaissances sur le CC peut s'écrire sur la forme du modèle économétrique (régression multiple) suivant :

(1) $\operatorname{ICC}_{\mathrm{ki}}=\beta_{0}+\beta_{1}(\text { DREC })_{i}+\beta_{2}(\text { STRUC })_{i}+\beta_{3}(\text { SEXE })_{i}+\beta_{4}(\text { AGE })_{i}$ $+\beta_{5}(\text { INTERN })_{\mathrm{i}}+\beta_{6}(\mathrm{PRO})_{\mathrm{i}}+\varepsilon_{\mathrm{i}}$

Pour comprendre la différence entre les « chercheurs » et les « expérimentateurs ", ce modèle peut s'écrire :

(2) $\mathrm{ICC}_{\mathrm{ki}}=\beta_{0}+\beta_{1}(\mathrm{DREC})_{\mathrm{i}}+\beta_{2}(\mathrm{STRUCF})_{\mathrm{i}}+\beta_{3}(\mathrm{SEXE})_{\mathrm{i}}+\beta_{4}(\mathrm{AGE})_{\mathrm{i}}$ $+\beta_{5}(\text { INTERN })_{\mathrm{i}}+\beta_{6}(\mathrm{PRO})_{\mathrm{i}}+\varepsilon_{\mathrm{i}}$

Pour isoler les « chercheurs » ce modèle s'écrit alors :

(3) $\operatorname{ICCR}_{\mathrm{ki}}=\beta_{0}+\beta_{1}(\mathrm{DREC})_{\mathrm{i}}+\beta_{2}(\mathrm{NAR})_{\mathrm{i}}+\beta_{3}(\mathrm{SEXE})_{\mathrm{i}}+\beta_{4}(\mathrm{AGE})_{\mathrm{i}}$ $+\beta_{5}(\text { INTERN })_{i}+\beta_{6}(\mathrm{PRO})_{\mathrm{i}}+\varepsilon_{\mathrm{i}}$

La proximité des acteurs de $R \& D$ avec les viticulteurs et les responsables de domaines ou de caves coopératives peut s'écrire sur la forme du modèle économétrique suivant (régression multiple) :

(4) CAVIX $_{\mathrm{ki}}=\beta_{0}+\beta_{1}(\text { DREC })_{\mathrm{i}}+\beta_{2}\left(\mathrm{STRUC}_{\mathrm{i}}+\beta_{3}(\mathrm{SEXE})_{\mathrm{i}}+\beta_{4}(\mathrm{AGE})_{\mathrm{i}}\right.$ $+\beta_{5}(\text { INTERN })_{i}+\beta_{6}(\text { RESO })_{i}++\varepsilon_{i}$

Pour comprendre la différence entre les « chercheurs » et les « expérimentateurs ", ce modèle peut s'écrire :

(5) CAVIX $_{\mathrm{ki}}=\beta_{0}+\beta_{1}(\text { DREC })_{\mathrm{i}}+\beta_{2}\left(\right.$ Strucf $_{\mathrm{i}}+\beta_{3}(\mathrm{SEXE})_{\mathrm{i}}+\beta_{4}(\mathrm{AGE})_{\mathrm{i}}$ $+\beta_{5}(\text { INTERN })_{\mathrm{i}}+\beta_{6}(\text { RESO })_{\mathrm{i}}++\varepsilon_{\mathrm{i}}$

Pour isoler les « chercheurs » ce modèle s'écrit alors :

(6) $\mathrm{CAVIR}_{\mathrm{ki}}=\beta_{0}+\beta_{1}(\mathrm{DREC})_{\mathrm{i}}+\beta_{2}(\mathrm{NAR})_{\mathrm{i}}+\beta_{3}(\mathrm{SEXE})_{\mathrm{i}}+\beta_{4}(\mathrm{AGE})_{\mathrm{i}}$ $+\beta_{5}(\text { INTERN })_{i}+\beta_{6}(\text { RESO })_{i}+\varepsilon_{i}$

Cependant pour tester l'effet de la région sur l'investissement des acteurs de la $R \& D$ dans la production de connaissances sur le CC et la proximité avec des acteurs économiques, nous avons tout simplement fait des régressions simples sur la variable Région, notée REG.

5. Certaines variables ne sont pas intégrées dans les modèles pour cause de robustesse car elles ne sont pas significatives. 


\section{Justification des variables}

Dans la littérature scientifique, de nombreux travaux abordent l'implication des chercheurs dans la sphère économique à partir des réseaux, des collaborations et des relations informelles entretenues avec des entreprises. Ces études mettent en évidence à la fois des caractéristiques des chercheurs et des facteurs systémiques (Giuliani et al., 2010). Certains travaux mettent l'accent sur le niveau d'éducation (Klofsten, Jones-Evans, 2000), le nombre de publications (Blumenthal et al., 1996; Landry et al., 2007), ou l'expérience à l'étranger. D'autres mettent en relief l'ancienneté, l'âge (D'Este, Patel, 2007 ; Boardman, Ponomariov, 2009) et le sexe (Gulbrandsen, Smeby, 2005; Van Rijnsoever et al., 2008). Une troisième catégorie de recherche met l'accent sur l'effet de la région (Cooke et al., 1997 ; Etzkowitz, Leydesdorff, 2000) et la structure de rattachement du chercheur (Mazzoleni, Nelson, 2007 ; Boardman, 2009). Enfin, nous suggérons que le domaine de recherche peut aussi influencer les relations entre chercheurs et entreprises. Nous supposons par exemple qu'un chercheur qui travaille sur des modèles mathématiques a une plus faible probabilité d'avoir des liens avec des entreprises viticoles que celui qui travaille sur la gestion du vignoble.

\section{RÉSULTATS}

\section{Chercheurs et expérimentateurs}

Les chercheurs intègrent plus l'enjeu CC dans leurs travaux que les expérimentateurs mais ont moins de relations avec les chefs d'entreprises vitivinicoles que ces derniers (modèles 2 et 5 ). Toutefois, au sein des expérimentateurs, une distinction doit être faite entre d'une part les Ingénieurs de l'IFV dont le niveau d'investissement dans le CC se rapproche de celui des chercheurs et, d'autre part, les agents des Chambres d'Agriculture et des interprofessions (Tableau 4). Il y a donc un effet lié à la position/fonction dans le Système d'Innovation, l'IFV apparaissant de ce point de vue plus proche de la recherche que du développement.

\section{Région d'exercice}

L'effet de la région joue de manière différente sur l'implication des acteurs de R\&D dans l'adaptation au CC (Tableaux 4 et 5) :

- Globalement, les acteurs de la R\&D du Languedoc-Roussillon et de Bordeaux s'investissent davantage dans la thématique du CC que ceux de Champagne. 
- Dans les trois régions, il n'y a pas de différence significative dans le niveau d'investissement des seuls chercheurs dans les travaux sur le CC. Il y a toutefois plus de chercheurs (en proportion) en LanguedocRoussillon et à Bordeaux, par rapport à la Champagne, qui font du CC l'un de leurs principaux axes de recherche.

En revanche en termes de proximité la tendance est différente :

- Il n'y a aucune différence significative entre les seuls chercheurs des différentes régions au sujet de leur proximité avec les viticulteurs ;

- Par contre, les acteurs de la $R \& D$ sont globalement plus proches des entreprises en Champagne qu'en Languedoc-Roussillon ou à Bordeaux.

\section{Domaine de recherche}

Les modèles économétriques montrent que le domaine de recherche influence de manière différente le niveau d'implication des acteurs de la $R \& D$ dans l'adaptation :

- Les chercheurs qui travaillent dans les domaines du matériel végétal et de la conduite du vignoble sont plus engagés dans la recherche sur le CC que ceux qui travaillent sur l'œnologie et la lutte contre les maladies;

- Les acteurs de la $R \& D$ qui travaillent sur le matériel végétal déclarent être plus impliqués dans la production de connaissances sur le CC que ceux qui travaillent sur la lutte contre les maladies, l'œnologie ou les stratégies spatiales et économiques. Ceux qui travaillent sur la conduite du vignoble déclarent s'intéresser beaucoup plus au CC que ceux qui travaillent sur la lutte contre les maladies.

On observe ici aussi une tendance différente pour les résultats sur la proximité :

- Les chercheurs qui travaillent sur les stratégies spatiales et économiques sont plus proches des entrepreneurs vitivinicoles que ceux qui travaillent sur la conduite du vignoble, la lutte contre les maladies ou le matériel végétal ;

- Plus globalement, les acteurs de la R\&D qui ont comme domaine de recherche les stratégies spatiales et économiques se démarquent par leur proximité avec les chefs d'entreprises vitivinicoles.

\section{Trajectoire et caractéristiques personnelles}

La trajectoire, les caractéristiques personnelles et l'excellence académique expliquent très peu l'implication du chercheur dans l'adaptation au 
changement climatique. Toutefois, le fait d'avoir participé à un projet de recherche sur le CC influence positivement le niveau d'investissement des acteurs de la $R \& D$ (modèles 1 à 3 ). Par ailleurs, les acteurs de la $R \& D$ qui ont fait un séjour à l'étranger sont moins proches des chefs d'entreprises que ceux qui ne l'ont pas fait (Tableau 4).

Tableau 4 - Statistiques descriptives, comparaison de moyenne par rapport aux variables ICC et CAVI pour les acteurs de la R\&D

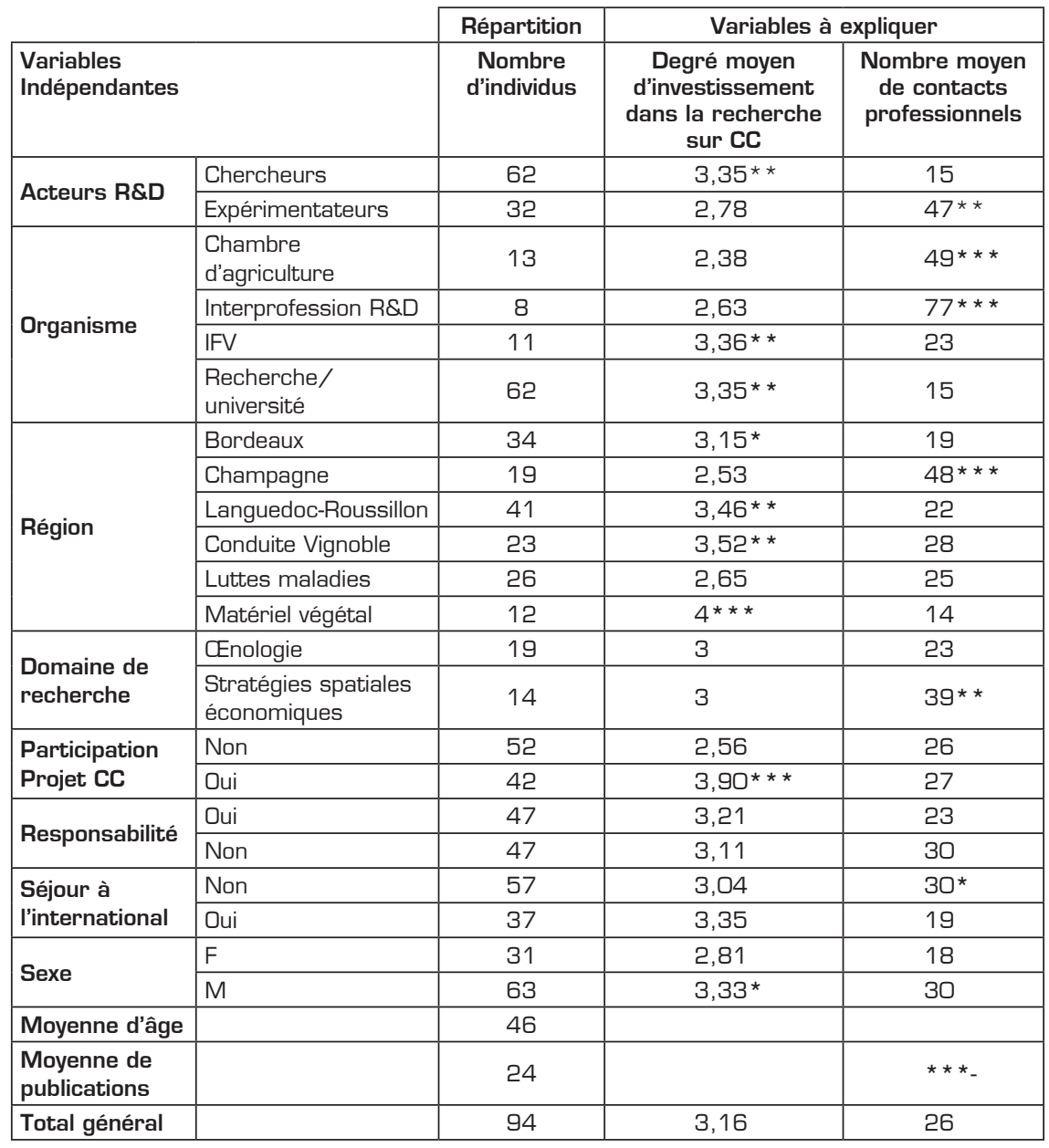

Source : J. Boyer, 2015

Significativité $10 \%$ *, Significativité $5 \%$ **, Significativité $1 \%$ ***

$\mathrm{Nb}$ : au niveau des variables ayant plusieurs modalités, la comparaison est faite par rapport à la modalité ayant la valeur la plus faible. 
Tableau 5 - Statistiques descriptives, comparaison de moyenne par rapport aux variables ICCREC et CAVIX pour les seuls chercheurs

\begin{tabular}{|c|c|c|c|c|}
\hline \multirow{2}{*}{\multicolumn{2}{|c|}{ Variables indépendantes }} & \multirow{3}{*}{\begin{tabular}{|c|} 
Répartition \\
$\begin{array}{c}\text { Nombre } \\
\text { d'individus }\end{array}$ \\
25 \\
\end{tabular}} & \multicolumn{2}{|c|}{ Variables à expliquer } \\
\hline & & & Degré moyen d'inves- & \\
\hline \multirow{3}{*}{ Région } & Bordeaux & & 3,40 & 17 \\
\hline & Champagne & 7 & 2,71 & 10 \\
\hline & Languedoc-Roussillon & 30 & 3,47 & 15 \\
\hline \multirow{5}{*}{$\begin{array}{l}\text { Domaine de } \\
\text { recherche }\end{array}$} & Conduite Vignoble & 14 & $3,79 * *$ & 8 \\
\hline & Luttes maladies & 15 & 2,80 & 11 \\
\hline & matériel végétal & 8 & $4,38 * * *$ & 9 \\
\hline & œnologie & 16 & 2,81 & 20 \\
\hline & $\begin{array}{l}\text { Stratégies spatiales } \\
\text { institutionnelles }\end{array}$ & 9 & $3,67 *$ & $32 * *$ \\
\hline \multirow{2}{*}{$\begin{array}{l}\text { Participation } \\
\text { Projet CC }\end{array}$} & Non & 31 & 2,45 & 13 \\
\hline & Oui & 31 & $4,26 * * *$ & 17 \\
\hline \multirow{2}{*}{ Sexe } & $\mathrm{F}$ & 22 & 2,95 & 9 \\
\hline & $M$ & 40 & 3,58 & 19 \\
\hline \multirow{2}{*}{ Responsabilité } & Non & 24 & 3,50 & 21 \\
\hline & Oui & 38 & 3,26 & 12 \\
\hline \multirow{2}{*}{$\begin{array}{l}\text { Séjour à } \\
\text { l'international }\end{array}$} & Oui & 31 & 3,45 & 14 \\
\hline & Non & 31 & 3,26 & 16 \\
\hline \multirow{3}{*}{$\begin{array}{l}\text { Niveau de } \\
\text { formation }\end{array}$} & Bac +5 et moins & 9 & 3,33 & 25 \\
\hline & Doctorat & 28 & 3,46 & 13 \\
\hline & HDR & 25 & 3,24 & 14 \\
\hline \multicolumn{2}{|c|}{ Nombre moyen de publications } & 34 & & \\
\hline \multicolumn{2}{|c|}{ Ancienneté dans la filière } & 20 & & \\
\hline \multicolumn{2}{|c|}{ Moyenne d'âge } & 48 & & \\
\hline \multicolumn{2}{|l|}{ Total } & 62 & 3,35 & 15 \\
\hline
\end{tabular}

Source : J. Boyer, 2015

Significativité $10 \%$ *, Significativité $5 \%$ **, Significativité $1 \% * * *$

NB : au niveau des variables ayant plusieurs modalités, la comparaison est faite par rapport à la modalité ayant la valeur la plus faible.

Tableau 6 - Résultats des modèles économétriques sur l'investissement des chercheurs

\begin{tabular}{|c|c|c|c|c|c|c|}
\hline \multirow{2}{*}{$\begin{array}{l}\text { Variables exp } \\
\text { strucf/Chercheur }\end{array}$} & \multicolumn{2}{|c|}{ Modèle 1} & \multicolumn{2}{|c|}{ Modèle 2} & \multicolumn{2}{|c|}{ Modèle 3} \\
\hline & $1.39 * * *$ & $1.39 * * *$ & $0.48 * *$ & $0.48 * *$ & & \\
\hline Ifv & $1.41 * * *$ & $1.41 * * *$ & & & & \\
\hline $\mathrm{Ca}$ & $0.94 *$ & $0.94 *$ & & & & \\
\hline \multicolumn{7}{|l|}{ Interpro } \\
\hline Mat & & 0.439 & & 0.57 & & 0.49 \\
\hline Eno & $-1.06 * * *$ & $-0.63 * *$ & $-1.17 * * *$ & $-0.60 *$ & $-1.24^{* * *}$ & $-0.75^{* *}$ \\
\hline Lut & $-0.93 * * *$ & $-0.50^{*}$ & $-1.13^{* * *}$ & $-0.56 * *$ & $-1.17 * * *$ & $-0.68 *$ \\
\hline
\end{tabular}




\begin{tabular}{|c|c|c|c|c|c|c|}
\hline Condt & -0.43 & & -0.57 & & -0.49 & \\
\hline Stratloc & $-1.16 * * *$ & $-0.73 * *$ & $-1.27 * * *$ & $-0.70 * *$ & $-1.20 * *$ & $-0.71 *$ \\
\hline Pro & $1.50 * * * *$ & $1.50 * * * *$ & $1.27 * * * *$ & $1.27 * * * *$ & $1.67 * \star \star *$ & $1.67 * * \star *$ \\
\hline Intern & -0.25 & -0.25 & -0.17 & -0.17 & -0.27 & -0.27 \\
\hline Age & 0.01 & 0.01 & 0.01 & 0.01 & 0.02 & 0.02 \\
\hline Sexe & 0.09 & 0.09 & 0.26 & 0.26 & 0.03 & 0.03 \\
\hline NAR & & & & & -0.01 & -0.01 \\
\hline _cons & 1.47 ** & $1.47^{* *}$ & 2.45 & 2.45 & 2.80 & 2.80 \\
\hline Number of obs & \multicolumn{2}{|l|}{94} & \multicolumn{2}{|l|}{94} & \multicolumn{2}{|l|}{62} \\
\hline R-squared & \multicolumn{2}{|l|}{0.49} & \multicolumn{2}{|l|}{0.44} & \multicolumn{2}{|l|}{0.57} \\
\hline Adj R-squared & \multicolumn{2}{|l|}{0.42} & \multicolumn{2}{|l|}{0.39} & \multicolumn{2}{|l|}{0.49} \\
\hline Prob $>$ F & \multicolumn{2}{|l|}{0.00} & \multicolumn{2}{|l|}{0.00} & \multicolumn{2}{|l|}{0.00} \\
\hline
\end{tabular}

Source : J. Boyer, 2015

Significativité $10 \% *$, Significativité $5 \% * *$, Significativité $1 \% * * *$, Significativité $0,1 \% * * * *$

Tableau 7 - Résultats des modèles économétriques sur la proximité avec les entreprises

\begin{tabular}{|c|c|c|c|c|c|}
\hline \multirow{2}{*}{$\begin{array}{l}\text { Variables exp } \\
\text { strucf/Chercheur }\end{array}$} & \multicolumn{3}{|c|}{ Modèle 4} & \multirow{2}{*}{$\begin{array}{c}\text { Modèle } 5 \\
-0.99 * * \star \star\end{array}$} & \multirow[t]{2}{*}{ Modèle 6} \\
\hline & $-1.52 * * * *$ & $-1.07 * * * *$ & $-0.65 * *$ & & \\
\hline Ifv & $-0.86 * *$ & -0.42 & & & \\
\hline $\mathrm{Ca}$ & -0.44 & & 0.42 & & \\
\hline Interpro & & 0.44 & $0.86 * *$ & & \\
\hline Mat & $-0.64^{*}$ & $-0.64^{*}$ & $-0.64 *$ & $-0.76 * \star$ & $-1.12^{* *}$ \\
\hline Eno & -0.25 & -0.25 & -0.25 & -0.30 & -0.50 \\
\hline Lut & $-0.64 * \star$ & $-0.64 * *$ & $-0.64 * *$ & $-0.66 * *$ & $-0.99 * *$ \\
\hline Condt & $-0.52^{*}$ & $-0.52^{*}$ & $-0.52^{\star}$ & $-0.54 *$ & $-1.09 * * \star$ \\
\hline \multicolumn{6}{|l|}{ Stratloc } \\
\hline RESO & $1.17 * \star \star \star$ & $1.17 * * * *$ & $1.17 * \star \star \star$ & $1.24 * * * *$ & $1.33 * * \star *$ \\
\hline INTERN & 0.12 & 0.12 & 0.12 & 0.09 & 0.21 \\
\hline Age & 0.01 & 0.01 & 0.01 & 0.01 & 0.02 \\
\hline Sexe & 0.17 & 0.17 & 0.17 & 0.09 & 0.03 \\
\hline NAR & & & & & -0.00 \\
\hline _cons & \multicolumn{3}{|c|}{$2.88 * * * *$} & $2.49 * * * *$ & $1.58 *$ \\
\hline Number of obs & \multicolumn{3}{|l|}{94} & 94 & 62 \\
\hline R-squared & \multicolumn{3}{|l|}{0.51} & 0.49 & 0.41 \\
\hline Adj R-squared & \multicolumn{3}{|l|}{0.45} & 0.43 & 0.31 \\
\hline Prob > F & \multicolumn{3}{|l|}{0.00} & 0.00 & 0.00 \\
\hline
\end{tabular}

Source : J. Boyer, 2015

Significativité $10 \% *$, Significativité $5 \%$ **, Significativité $1 \% * * *$, Significativité $0,1 \% * * * *$ 


\section{DISCUSSION}

\section{Dualité entre investissement dans la recherche et proximité avec les acteurs}

Les résultats révèlent une diversité de profils d'acteurs de la $R \& D$ au regard d'une part de leur investissement dans la production de connaissances sur le CC et, d'autre part, de leurs liens avec les viticulteurs. Certains sont à l'écart de ces deux enjeux ; d'autres intègrent fortement le CC dans leurs travaux mais ont peu de liens avec les viticulteurs ou vice et versa. Pour l'ensemble des acteurs étudiés, ces deux composantes de la capacité d'adaptation apparaissent de fait indépendantes, mais par contre plusieurs facteurs influencent cette dualité.

\section{Une déclinaison différente selon les vignobles régionaux}

Tout d'abord, la localisation régionale des acteurs de la $R \& D$ joue sur leur investissement dans la recherche et leurs liens avec les acteurs économiques, avec une distinction entre d'un côté la Champagne (moins concernée par le CC, mais présentant plus de liens), et de l'autre côté le Languedoc-Roussillon et Bordeaux. Cette dualité peut être expliquée par le fait que les conséquences du $\mathrm{CC}$ diffèrent selon les régions en nature, en intensité et en fréquence (IPCC, 2014). La région de Champagne bénéficie jusqu'à présent d'effets positifs du CC avec une diminution de coûts œnologiques, une amélioration des rendements et une fréquence accrue de bons millésimes (Quénol, 2014). A l'inverse, à Bordeaux comme en LanguedocRoussillon, les acteurs voient davantage le CC comme une "menace» en particulier sur les qualités du vin. Le débat sur le CC y est de ce fait davantage présent, de nombreux chercheurs s'étant impliqués dans le projet LACCAVE ${ }^{6}$ de l'INRA et contribuent à révéler l'impact du CC. De son côté, le vignoble de Champagne bénéficie d'un comité interprofessionnel (CIVC) qui, non seulement s'implique directement dans la $R \& D$, mais joue aussi un rôle d'intermédiaire, de médiation entre la recherche et les viticulteurs pour d'autres thèmes que le CC (Panigai et al., 2014). L'effet régional exprime donc des différences d'exposition au CC et d'organisation héritée de la $R \& D$ pour le vin.

6. Le projet LACCAVE (Long term Adaptation to Climate ChAnge in Viticulture and Enology) est un projet de recherche qui mobilise une centaine de chercheurs. Il est issu du métaprogramme ACCAF (Adaptation to Climate Change for Agrosystems and Forests) de l'INRA. 


\section{L'effet des domaines de recherche}

Les acteurs de la $R \& D$ qui travaillent sur le matériel végétal et ceux qui travaillent sur les stratégies spatiales et économiques illustrent aussi la dualité d'influence des domaines de recherche. Les premiers se distinguent par un fort investissement dans la recherche sur le CC mais ont peu de liens avec les viticulteurs, alors que les autres ont à l'inverse un plus faible investissement et beaucoup de liens. Les domaines de recherche ont de fait des rapports différents vis-à-vis de la question du CC. La recherche sur le matériel végétal s'organise autour de la génétique, une science fondamentale. Elle est vue comme un levier important de l'adaptation à long terme (Ollat, Touzard, 2014), en sélectionnant des cépages résistants à la sècheresse, plus tardifs ou produisant moins de sucre. Les chercheurs doivent alors intégrer des « critères de sélection » qui prennent en compte les conditions de production à l'horizon d'une cinquantaine d'années, du fait de la durée du processus de sélection (une quinzaine d'années) et de la durée de vie d'une plantation (une quarantaine d'années). Il s'agit de l'horizon des scénarios du CC. En revanche, les acteurs de la $R \& D$ qui travaillent sur les stratégies économiques et spatiales sont interpellés par les préoccupations à court-terme des viticulteurs, sur les revenus, les pratiques, les marchés, l'exportation des vins, l'enjeu climatique étant souvent secondaire par rapport à celui de l'adaptation à la demande. De plus ils côtoient des responsables d'entreprises pour la réalisation d'enquêtes ou d'entretiens, une de leurs méthodes de recherche (Neuman, 2005; Denzin, Lincoln, 2009). Chaque domaine de recherche est donc concerné par des méthodes, des pertinences et des horizons différents vis-à-vis du CC, ce qui est d'ailleurs un argument justifiant la place de l'interdisciplinarité dans les programmes de recherche sur le CC (Morin, 1994).

\section{Construction scientifique ou pratique de l'enjeu climatique}

Nos résultats renvoient aussi au fait que le CC est un enjeu principalement construit par la recherche, à partir d'observations scientifiques au niveau mondial, animé par le GIEC (Groupe d'experts intergouvernemental sur l'évolution du climat). Les innovations qui en découlent suivent, à un certain niveau, un modèle d'innovation de type top-down (Cronin, 2014), très présent dans la notion de Climate Smart Agriculture (FAO, 2010). L'origine scientifique de la construction de l'enjeu du CC peut expliquer pourquoi les «chercheurs » intègrent beaucoup plus cet enjeu dans leurs travaux que les « expérimentateurs ». Ce résultat est aussi en accord avec le rapport temporel entre la recherche fondamentale et la recherche appliquée ou le développement des produits innovants. Le « nouvel enjeu » du CC est 
donc approprié d'abord par la recherche fondamentale, qui fournit des résultats pour des acteurs et organismes chargés d'expérimentation et de développement. A l'opposé ces derniers (en particulier Chambres d'Agriculture et interprofessions) sont plus en contact avec les entreprises, impliqués dans une construction pratique et locale de l'enjeu climatique, souvent très relatif par rapport à d'autres préoccupations notamment économiques (Bostrom et al., 1994 ; Dollfus, 2001 ; Lorius, 2003). Cette dualité peut aussi expliquer pourquoi les acteurs de la $R \& D$ sont plus engagés dans la recherche sur le $\mathrm{CC}$ en Languedoc-Roussillon et à Bordeaux - deux régions qui concentrent l'essentiel de la recherche publique sur la vigne et le vin en France - qu'en Champagne.

\section{Système Sectoriel d'Innovation et capacité d'adaptation}

L'analyse des facteurs qui jouent sur la contribution des acteurs de la $R \& D$ à l'adaptation renforce l'intérêt des approches en termes de Système Sectoriel d'Innovation, en soulignant l'importance de l'agencement interne des acteurs de ce système et les relations qu'ils entretiennent.

\section{L'agencement du Système Sectoriel d'Innovation}

Nos résultats mettent en lumière une dualité de l'ensemble des acteurs de la $R \& D$ par rapport à leur investissement dans la production de connaissances sur le CC et leur capacité à tisser des liens avec les viticulteurs, en particulier en fonction de leur région d'exercice. Cette différence n'est plus significative dès lors que l'on isole le groupe des seuls " chercheurs ", qui ont en moyenne des profils proches dans les trois régions. Tout se joue donc selon l'agencement du Système d'Innovation vitivinicole au niveau régional. Les organismes, comme les centres de recherche publique et les universités, sont plus impliqués dans les travaux sur le CC mais sont moins proches des viticulteurs que les Chambres d'Agriculture et les interprofessions, ce qui est cohérent avec les "missions » attribuées à chaque type d'organisme. C'est bien la manière dont elles vont se combiner, se compléter dans chaque Système Régional d'Innovation, qui compte et peut jouer sur l'innovation dans les vignobles. Dans cet agencement nos travaux indiquent la place centrale que peut jouer une interprofession (exemple de la Champagne) capable de structurer une demande à destination des acteurs de la $R \& D$ en région et hors région (Touzard, 2011). L'enjeu est de concilier les deux principales fonctions des Systèmes d'Innovation : la disponibilité des connaissances en rapport avec le CC et leur accessibilité (Jacobsson, Johnson, 2000). 


\section{La dimension relationnelle des Systèmes Sectoriels d'Innovation}

Le concept de réseaux occupe une place centrale dans les approches en termes de Système Sectoriel d'innovation (Edquist, 1997 ; Malherba, 2002). Si dans les vignobles régionaux du Languedoc-Roussillon et de Bordeaux, les acteurs de la $R \& D$ s'investissent plus dans la recherche sur le CC, des travaux empiriques ont pourtant montré que les viticulteurs de Champagne se sentent autant concernés par l'enjeu changement climatique, sont capables de traduire ses conséquences sur différents domaines d'action, et innovent autant que ceux du Languedoc-Roussillon ou de Bordeaux (Jouan, 2014). Le vignoble champenois jouit d'un capital relationnel important. Pas moins de 7 réseaux formels, qui traitent des enjeux vitivinicoles différents, coordonnés par le CIVC ont par exemple été répertoriés (Panigai et al., 2014). Ceux-ci sont déterminants pour la capacité d'adaptation des viticulteurs et maisons de Champagne, pour des enjeux qui certes ne mettent pas (encore) en avant le CC, mais pourraient rapidement le faire. L'existence de réseaux formels et informels est bien au cœur d'un Système d'Innovation (Touzard, 2014), permettant coopération, innovation et réactivité dans une région viticole donnée, et les caractéristiques de ces réseaux (leur structure en particulier) peuvent apparaître au final plus importantes que le « concernement » (awareness) sur un enjeu particulier, évolutif (Chiffoleau, Touzard, 2014). L'analyse des réseaux de partenariats entre chercheurs et entreprises au sein d'un Système d'Innovation, à partir des liens de conseil ou autres, est donc une voie importante pour mieux comprendre et appuyer le processus d'adaptation au CC.

\section{Caractéristiques personnelles et excellence académique}

Contrairement à d'autres travaux (D'Este, Patel 2007; Boardman, Ponomariov 2009 ; Giuliani et al., 2010), nos résultats empiriques ne permettent pas de confirmer le rôle des caractéristiques personnelles et de l'excellence académique des acteurs de la $R \& D$ sur leur proximité avec les entreprises, et encore moins sur leur investissement dans la production de connaissances sur le CC. Trois raisons peuvent expliquer cette opposition apparente entre nos résultats et ceux de la littérature. Tout d'abord les entreprises étudiées sont des TPE et PME, sans service de $R \& D$, qui s'impliquent peu dans des projets de recherche et d'expérimentation avec des chercheurs et n'ont pas forcément accès aux publications scientifiques. Ces caractéristiques sont une justification à l'existence de «brokers » et d'organisations intermédiaires de $R \& D$ (Chambres d'Agriculture, Interprofessions...) qui atténueraient en quelque sorte l'importance des caractéristiques personnelles 
des chercheurs pour l'adaptation. En second lieu, les chercheurs sont évalués sur des publications scientifiques sans prendre en compte leurs impacts sur les entreprises, et encore moins celles de leur région, ce qui ne crée pas d'incitation à développer l'excellence académique ou d'autres caractéristiques personnelles dans l'engagement local pour l'adaptation au CC. Giuliani et al. (2010) avait d'ailleurs noté une corrélation négative entre l'excellence académique d'un universitaire et ses contributions directes à l'innovation dans le vignoble de Western Cape (Giuliani et al., 2010). Enfin, l'enjeu du CC est encore récent et n'est sans doute pas encore un domaine de recherche suffisamment constitué pour différencier les chercheurs par rapport à leurs caractéristiques personnelles.

\section{CONCLUSION}

Cette étude analyse l'implication des acteurs de la $R \& D$ dans l'adaptation des entreprises vitivinicoles (domaines, caves coopératives) au CC et sous quelles conditions. Nous avons développé le concept «implication » à partir d'une analyse de l'investissement du chercheur dans la production de connaissances sur le changement climatique, et de ses relations avec les entreprises. En partant d'enquêtes dans trois vignobles, ces deux dimensions de l'adaptation ont été approchées par deux variables synthétiques, puis analysées par rapport à des facteurs qui renvoient aux caractéristiques personnelles du chercheur, à son excellence académique, à son domaine de recherche, à son organisme de rattachement et à sa région d'exercice.

Cette recherche met en évidence une dualité. En effet, la région d'exercice, le domaine de recherche et l'organisation de rattachement influencent de manière différente l'investissement des acteurs de la $R \& D$ dans la production de connaissances sur le CC et leur proximité avec les acteurs économiques. En revanche, l'excellence académique et les caractéristiques personnelles ne jouent pas sur cette implication. Il ressort que l'investissement du chercheur dans la production de connaissances autour du CC n'est donc pas suffisant pour aider les entreprises à s'adapter au CC. Son engagement dans le processus de transfert, sa proximité avec les entreprises et les réseaux mobilisables jouent un rôle clé pour améliorer la capacité des entreprises à faire face au changement climatique. Ces résultats renforcent l'importance des projets de recherche interdisciplinaires sur l'adaptation au CC et d'une réflexion stratégique sur l'agencement des Systèmes d'Innovation.

Le cadre d'analyse des Systèmes Sectoriels d'Innovation apparaît alors pertinent pour étudier les conditions institutionnelles, cognitives et relationnelles de l'adaptation au CC. L'enjeu scientifique consiste alors à analyser de 
manière plus approfondie les relations entre l'agencement des institutions de $R \& D$, la production de connaissances pour l'adaptation et la dimension relationnelle des Systèmes Sectoriels d'Innovation. Celle-ci peut être d'une grande utilité pour fournir un éclairage théorique et empirique sur le rôle de la recherche dans l'adaptation au CC. Elle peut aussi aider les responsables politiques et professionnels dans l'élaboration des politiques de recherche et d'innovation et la mobilisation des leviers d'adaptation possibles, en particulier pour les vignobles régionaux. L'importance des incertitudes liées au CC amène à raisonner de plus en plus en termes de « capacité d'adaptation » et il apparaît que, pour cela, la coordination régionale de réseaux de coopération entre acteurs de la $R \& D$ et entreprises soit plus importante que l'état de « concernement » des enjeux eux-mêmes entre des acteurs au sein d'une même région.

\section{BIBLIOGRAPHIE}

BlumEnthal, D., CAMPBEll, E. G., CAUSinO N., LOUIS, K. S. (1996), Participation of Life-Science Faculty in Research Relationships with Industry, New England journal of medicine, 335(23), 1734-1739.

BOARDMAN, P. C. (2009), Government Centrality to University-Industry Interactions: University Research Centers and the Industry Involvement of Academic Researchers, Research Policy, 38(10), 1505-1516.

BOARDMAN, P. C., PONOMARIOV, B. L. (2009), University Researchers Working with Private Companies, Technovation, 29(2), 142-153.

BOSTROM, A., MORGAN, M., FISCHHOFF, B., READ, D. (1994), What do People Know about Global Climate Change? 1. Mental Models, Risk Analysis, 14(6), 959-970.

BOYER, J., TEMPLE, L., SCUTT, R. (2014), Etude de cas : la technique Minisett en Haïti, une innovation co-construite entre chercheurs et producteurs dans le respect des conditions locales et des enjeux agro-écologiques, Field Actions Science Reports, 9, Spécial Issue. BRICHE, É. (2011), Changement climatique dans le vignoble de Champagne: Modélisation thermique à plusieurs échelles spatio-temporelles (1950-2100), Université Paris-Diderot-Paris VII. CARBONNEAU, A., OJEDA, H. (2012), Ecophysiologie et gestion de l'eau en viticulture, Le Progrès Agricole et Viticole, 129(21), 508-512.

CHIFFOLEAU, Y., TOUZARD, J. M. (2014), Understanding Local Agri-Food Systems through Advice Network Analysis, Agriculture and Human Values, 31(1), 19-32.

COHEN, W. M., NELSON, R. R., WALSH, J. P. (2002), Links and Impacts: The Influence of Public Research on Industrial R\&D, Management Science, 48(1), 1-23.

COOKE, P., URANGA, M., GOMEZ, M., ETXEBARRIA, G. (1997), Regional Innovation Systems: Institutional and Organisational Dimensions, Research Policy, 26, 475-491.

CRAMER, W., BONDEAU, A., WOODWARD, F. I., PRENTICE, I. C., BETTS, R. A., BROVKIN, V., COX, P. M., FISHER, V., FOLEY, J. A., FRIEND, A. D., KUCHARIK, C., 
LOMAS, M. R., RAMANKUTTY, N., SITCH, S., SMITH, B., WHITE, A.,YOUNGMOLLING, C. (2001), Global response of Terrestrial Ecosystem Structure and Function to $\mathrm{CO} 2$ and Climate Change: Results from Six Dynamic Global Vegetation Models, Global Change Biology, 7, 357-373.

CRONIN, M., J. (2014), Top Down Innovation, New York, Springer.

D'ESTE, P., PATEL, P. (2007), University - Industry Linkages in the UK: What are the Factors Underlying the Variety of Interactions with Industry?, Research Policy, 36(9), $1295-1313$.

DENZIN, N. K., LINCOLN, Y. S. (2009), Qualitative Research, Yogyakarta, PustakaPelajar. DOLlFUS, O. (2001), La mondialisation, Paris, Presses de sciences po.

DUCHÊNE, E., HUARD, F., DUMAS, V., SCHNEIDER, C., MERDINOGLU, D. (2010), The Challenge of Adapting Grapevine Varieties to Climate Change, Climate research, 41(3), 193-204.

EDQUIST, C. (1997), Systems of Innovation: Technologies, Institutions, and Organizations, Psychology Press.

ETZKOWITZ, H., LEYDESDORFF, L. (2000), The Dynamics of Innovation: From National Systems and "Mode 2" to a Triple Helix of University-Industry-Government Relations, Research Policy, 29, 109-123.

FAO (2010), Climate-Smart Agriculture: Policies, Practice and Financing for Food Security, Adaptation and Migration.

FÜSSEL, H. M. (2007), Adaptation planning for Climate Change: Concepts, Assessment Approaches, and Key Lessons, Sustainability Science, 2(2), 265-275.

GHINI, R., HAMADA, E., ANGELOTTI, F., COSTA, L. B., BETTIOL, W. (2012), Research Approaches, Adaptation Strategies, and Knowledge Gaps Concerning the Impacts of Climate Change on Plant Diseases, Tropical Plant Pathology, 37(1), 5-24.

GIULIANI, E., ARZA, V. (2009), What Drives the Formation of 'Valuable' University Industry Linkages?, Research Policy, 38(6), 906-921.

GIULIANI, E., BELL, M. (2005), The Micro-Determinants of Meso-Level Learning and Innovation: Evidence from a Chilean Wine Cluster, Research Policy, 34(1), 47-68.

GIULIANI, E., MORRISON, A., PIETROBELLI, C., RABELLOTTI, R. (2010), Who are the Researchers that are Collaborating With Industry? An Analysis of the Wine Sectors in Chile, South Africa and Italy, Research Policy, 39(6), 748-761.

GOUTOULY, J. P. (2009), Impacts du changement climatique sur la vigne et le raisin, Revue française d'oenologie, 235, 23-26.

GRIN, J. (2010), Acting Locally, Developing Knowledge Globally: A Transitions Perspective on Designing Climate Change Adaptation Strategies, in Driessen, J., Leroy, P., Van Vierssen, W. (eds), From Climate Change to Social Change: Perspectives on Science-Policy Interactions, Utrecht, International Books, 95-108.

GULBRANDSEN, M., SMEBY, J.-C. (2005), Industry Funding and University Professors' Research Performance, Research Policy, 34(6), 932-950.

HANNAH, L., ROEHRDANZ, P. R., IKEGAMI, M., SHEPARD, A. V., SHAW, M. R., TABOR, G., ZHI, L., MARQUET, P. A., HIJMANS, R. J. (2013), Climate Change, Wine, and Conservation, Proceedings of the National Academy of Sciences, 110(17), 6907-6912. 
HINNEWINKEL, J. C. (2007), Le vignoble bordelais à l'épreuve du changement de climat, Colloque Réchauffement climatique, quels impacts probables sur les vignobles, Dijon Beaune, 28-30 mars 2007.

HOLlAND, T., SMIT, B. (2010), Climate Change and the Wine Industry: Current Research Themes and New Directions, Journal of Wine Research, 21(2/3), 125-136.

IPCC (2001), Climate Change 2001: Impacts, Adaptation, and Vulnerability: Contribution of Working Group II to the Third Assessment Report of the Intergovernmental Panel on Climate Change, Cambridge, Cambridge University Press.

IPCC (2014), Climate Change 2014: Synthesis Report. Contribution of Working Groups I, II and III to the Fifth Assessment Report of the Intergovernmental Panel on Climate Change, Cambridge, Cambridge University Press

JACOBSSON, S., JOHNSON, A. (2000), The Diffusion of Renewable Energy Technology: An Analytical Framework and Key Issues for Research, Energy Policy, 28(9), 625-640.

JONES, G. V. (2007), Climate Change: Observations, Projections, and General Implications for Viticulture And Wine Production, Economics department working paper, 7(1).

JOUAN, J. (2014), Les AOC viticoles face au changement climatique: Exploration des voies d'adaptation par la prospective et l'analyse économique, Thèse de Doctorat, Agrocampus Ouest KLOFSTEN, M., JONES-EVANS, D. (2000), Comparing Academic Entrepreneurship in Europe: The Case of Sweden and Ireland, Small Business Economics, 14(4), 299-309.

KONTOUDAKIS, N., ESTERUELAS, M., FORT, F., CANALS, J. M., DE FREITAS, V., ZAMORA, F. (2011), Influence of the Heterogeneity of Grape Phenolic Maturity on Wine Composition and Quality, Food Chemistry, 124(3), 767-774.

LANDRY, R., AMARA, N., OUIMET, M. (2007), Determinants of Knowledge Transfer: Evidence from Canadian University Researchers in Natural Sciences and Engineering, The Journal of Technology Transfer, 32(6), 561-592.

LE TREUT, H. (2003), Global Scenarios of Climate Change and Associated Uncertainties, Comptes rendus Géoscience, Académie des Sciences, Paris, 335(6), 525-533.

LEARY, N., BAETHGEN, M., BARROS, V., BURTON, I., CANZIANI, O., DOWNING, T. E., KLEIN, R., MALPEDE, D., MARENGO, J. A., MEARNS, L. O. (2006), A Plan of Action to Support Climate Change Adaptation Through Scientific Capacity, Knowledge and Research, AIACC Report, 23.

LORIUS, C. (2003), Effet de serre: les lacunes du savoir et de la perception, Comptes Rendus Géoscience, Académie des Sciences, Paris, 335(6), 545-549.

MALERBA, F. (2002), Sectoral Systems of Innovation and Production, Research Policy, 31, 247-64.

MAZZOLENI, R., NELSON, R. R. (2007), Public Research Institutions and Economic Catch-Up, Research Policy, 36(10), 1512-1528.

MORIN, E. (1994), Sur l'interdisciplinarité, Bulletin interactif du centre international de recherches et études transdisciplinaires, 2(2).

NEUMAN, W. L. (2005), Social Research Methods: Quantitative and Qualitative Approaches, Boston, Allyn and Bacon.

NYONG, A., ADESINA, F., ELASHA,B. O. (2007), The Value of Indigenous Knowledge in Climate Change Mitigation and Adaptation Strategies in the African Sahel, Mitigation and Adaptation Strategies for Global Change, 12(5), 787-797. 
OLLAT, N., TOUZARD, J. M. (2014), Adaptation à long terme au changement climatique pour la viticulture et l'œnologie : un programme de recherche sur les vignobles français, Revue des oenologues et des techniques vitivinicoles et oenologiques, 41(152), 11-12.

PANIGAI, L., VACAVANT, M. P., MONCOMBLE, D. (2014), Les réseaux en viticulture. Une pépite méconnue de la Champagne, Le Vigneron champenois, 135(4), 39-61.

PENNESI, K., AROKIUM, J., MCBEAN, G. (2012), Integrating Local and Scientific Weather Knowledge as a Strategy for Adaptation to Climate Change in the Arctic, Mitigation and Adaptation Strategies for Global Change, 17(8), 897-922.

QUÉNOL, H. (2014), Changement climatique et terroirs viticoles, Paris, Lavoisier Tec\&doc. REEDY, D., SAVO, V., MCCLATCHEY, W. (2014), Traditional Climatic Knowledge: Orchardists' Perceptions of and Adaptation to Climate Change in the Campania Region (Southern Italy), Plant Biosystems-An International Journal Dealing with all Aspects of Plant Biology, 148(4), 699-712.

RODRIGO, C. (2015), Evaluating the Adaptive Capacity to Climate Change: A Case from Rural Agriculture of Sri Lanka, Wudpecker Journal of Agricultural Research, 4(1), 1-5.

ROSENZWEIG, C., WILBANKS, T. J. (2010), The State of Climate Change Vulnerability, Impacts, and Adaptation Research: Strengthening Knowledge Base and Community, Climatic Change, 100(1), 103-106.

SANTHA, S. D., FRAUNHOLZ, B., UNNITHAN, C. (2010), A Societal Knowledge Management System: Harnessing Indigenous Wisdom to Build Sustainable Predictors for Adaptation to Climate Change, International journal of climate change: impacts and responses, 2(1), 49-64.

SCHIPPER, E. L. F., BURTON, I. (2009), Understanding Adaptation: Origins, Concepts, Practice and Policy, in Schipper, E. L. F., Burton, I. (eds), The Earthscan Reader on Adaptation to Climate Change, London, Earthscan.

SCHUlTZ, H. (2000), Climate Change and Viticulture: A European Perspective on Climatology, Carbon Dioxide and UV-B Effects, Australian Journal of Grape and Wine Research, 6(1), 2-12.

SCHULTZ, H., STOLL, M. (2010), Some Critical Issues in Environmental Physiology of Grapevines: Future Challenges and Current Limitations, Australian Journal of Grape and Wine Research, 16(1), 4-24.

SMIT, B., PILIFOSOVA, O. (2003), Adaptation to Climate Change in the Context of Sustainable Development and Equity, Sustainable Development, 8(9), 9.

SMIT, B., WANDEL, J. (2006), Adaptation, Adaptive Capacity and Vulnerability, Global Environmental Change, 16(3), 282-292.

STERN, N. H., TREASURY, H. M. S. (2006), Stern Review: The Economics of Climate Change, London, HM treasury.

TOUZARD, J. M. (2011), Les réseaux professionnels : facteur clé pour l'innovation dans le secteur vitivinicole?, Revue française d'oenologie, 249, 47-50.

TOUZARD, J. M. (2014), Les approches sectorielles de l'innovation, in Boutillier, S., Forest, J., Gallaud, D., Laperche, B., Tanguy, C., Temri, L. (dir.), Principes d'économie de l'innovation, Bruxelles, Peter Lang, 235-245.

VAN LEEUWEN, C., SCHULTZ, H. R., DECORTAZAR-ATAURI, I. G., DUCHÊNE, E., OllAT, N., PIERI, P., BOIS, B., GOUTOUlY, J. P., QUÉNOL, H., TOUZARD, J. M. 
(2013), Why Climate Change will not Dramatically Decrease Viticultural Suitability in Main Wine-Producing Areas by 2050, Proceedings of the National Academy of Sciences, 110(33), 3051-3052.

VAN RIJNSOEVER, F. J., HESSELS, L. K., VANDEBERG, R. L. (2008), A ResourceBased View on the Interactions of University Researchers, Research Policy, 37(8), $1255-1266$.

VESCOVI, L., BOURQUE, A., SIMONET, G., MUSY, A. (2009), Transfer of Climate Knowledge via a Regional Climate-Change Management Body to Support Vulnerability, Impact Assessments and Adaptation Measures, Climate Research, 40(23), 163-173. 